University of Nebraska - Lincoln

DigitalCommons@University of Nebraska - Lincoln

Faculty Publications in Computer \& Electronics Electrical \& Computer Engineering, Department Engineering (to 2015)

2005

\title{
A Generalized Algorithm for the Generation of Correlated Rayleigh Fading Envelopes
}

\author{
Le Chung Tran \\ University of Wollongong \\ Tadeusz Wysocki \\ University of Nebraska-Lincoln, wysocki@uow.edu.au \\ Jennifer Seberry \\ University of Wollongong \\ Alfred Mertins \\ University of Oldenburg
}

Follow this and additional works at: https://digitalcommons.unl.edu/computerelectronicfacpub

Part of the Computer Engineering Commons

Tran, Le Chung; Wysocki, Tadeusz; Seberry, Jennifer; and Mertins, Alfred, "A Generalized Algorithm for the Generation of Correlated Rayleigh Fading Envelopes" (2005). Faculty Publications in Computer \& Electronics Engineering (to 2015). 38.

https://digitalcommons.unl.edu/computerelectronicfacpub/38

This Article is brought to you for free and open access by the Electrical \& Computer Engineering, Department of at DigitalCommons@University of Nebraska - Lincoln. It has been accepted for inclusion in Faculty Publications in Computer \& Electronics Engineering (to 2015) by an authorized administrator of DigitalCommons@University of Nebraska - Lincoln. 


\section{A Generalized Algorithm for the Generation of Correlated Rayleigh Fading Envelopes}

\author{
Le Chung Tran, Tadeusz A. Wysocki, Jennifer Seberry \\ School of Elec., Comp. and Telecom. Eng. \\ University of Wollongong \\ Northfields Avenue, NSW 2522, Australia \\ \{lct71,wysocki,jennie\}@uow.edu.au
}

\author{
Alfred Mertins \\ Institute of Physics \\ University of Oldenburg \\ 26111 Oldenburg, Germany \\ alfred.mertins@uni-oldenburg.de
}

\begin{abstract}
Although generation of correlated Rayleigh fading envelopes has been intensively considered in the literature, all conventional methods have their own shortcomings, which seriously impede their applicability. In this paper, a very general, straightforward algorithm for generation of an arbitrary number of Rayleigh envelopes with any desired, equal or unequal power, in wireless channels either with or without Doppler frequency shifts, is proposed. The proposed algorithm can be applied in case of spatial correlation, such as with antenna arrays in Multiple Input Multiple Output (MIMO) systems, or spectral correlation between the random processes like in Orthogonal Frequency Division Multiplexing (OFDM) systems. It can also be used for generating correlated Rayleigh fading envelopes in either discrete-time instants or a real-time scenario. Besides being more generalized, our proposed algorithm is more precise, while overcoming all shortcomings of the conventional methods.
\end{abstract}

\section{Introduction}

Generation of correlated Rayleigh fading envelopes has been intensively examined in the literature. However, all conventional methods have their own shortcomings, which seriously limit their applicability. To point this out more clearly, we first analyze the shortcomings of some interesting conventional methods.

In [6], the authors derived fading correlation properties in antenna arrays and, then, briefly mentioned the algorithm to generate complex Gaussian random variables (with Rayleigh envelopes) corresponding to a desired correlation coefficient matrix. This algorithm was proposed for generating equal power Rayleigh envelopes only, rather than arbitrary (equal or unequal) power Rayleigh envelopes.

In the papers [3] and [1], the authors proposed different methods for generating only $N=2$ equal power correlated Rayleigh envelopes. In [2], the authors generalized the method in [1] for $N \geq 2$. However, in this method, Cholesky decomposition is used, and consequently, the covariance matrix must be positive definite, which is not always realistic.

These methods were then more generalized in [5], where one can generate any number of Rayleigh envelopes corresponding to a desired covariance matrix and with any power, i.e., even with unequal power. However, again, the covariance matrix must be positive definite in order for Cholesky decomposition to be performable. In addition, the authors in [5] forced the covariances of the complex Gaussian random variables (with Rayleigh fading envelopes) to be real (see Eq. (8) in [5]). This limitation is incorrect in various cases because, in fact, the covariances of the complex Gaussian random variables are more likely to be complex.

In [7], the authors proposed a method for generating any number of Rayleigh envelopes with equal power only. Although the method in [7] works well in various cases, it however fails to perform Cholesky decomposition for some complex covariance matrices in MatLab due to the roundoff errors of Matlab. This shortcoming is overcome by some modifications mentioned later in our proposed algorithm.

More importantly, the method proposed in [7] fails to generate Rayleigh fading envelopes corresponding to a desired covariance matrix in a real-time scenario where Doppler frequency shifts are considered. This is because passing Gaussian random variables with variances assumed to be unit (for simplicity of explanation) through a Doppler filter changes remarkably the variances of those variables. The variances of the variables at the outputs of Doppler filters are not unit any more, but are arbitrary depending on the variance of the variables at the inputs of the filters as well as the characteristics of those filters. The authors in [7] did not realize this variance-changing effect caused by Doppler filters. We will return this issue later in this paper.

For these reasons, a more generalized algorithm which overcomes all shortcomings of the conventional methods and is applicable to various scenarios is required to be considered. This paper proposes one of such algorithms.

The paper is organized as follows. In Sections 2, we shortly review the discussions on the correlation property between the transmitted signals as functions of time delay and frequency separation, such as in OFDM systems, and as functions of spatial separation between transmission antennas, such as in MIMO systems. In Section 3, we propose a very general, straightforward algorithm to generate correlated Rayleigh fading envelopes. Section 4 derives an algorithm to generate correlated Rayleigh fading envelopes in a real-time scenario. Simulation results are presented in Section 5. The paper is concluded by Section 6 . 


\section{Fading correlation as functions of fre- quency separation as well as of spatial sep- aration}

In [4], Jakes considered the scenario where all complex Gaussian random processes with Rayleigh envelopes have equal powers $\sigma^{2}$ and derived the correlation properties between random processes as functions of both time delay and frequency separation, such as in OFDM systems. Let $z_{k}(t)$ and $z_{j}(t)$ be the two zero-mean complex Gaussian random processes at time instant $t$, corresponding to frequencies $f_{k}$ and $f_{j}$, respectively. Denote $x_{k}$ and $y_{k}$ to be the real and imaginary parts of $z_{k}(t)$, while $x_{j}$ and $y_{j}$ the real and imaginary parts of $z_{j}\left(t+\tau_{k, j}\right)$, where $\tau_{k, j}$ is the arrival time delay between two signals at the considered time instant. By definition, the covariances between the real and imaginary parts of $z_{k}(t)$ and $z_{j}\left(t+\tau_{k, j}\right)$ are: $R_{x x k, j} \triangleq E\left(x_{k} x_{j}\right)$; $R_{y y_{k, j}} \triangleq E\left(y_{k} y_{j}\right) ; R_{x y_{k, j}} \triangleq E\left(x_{k} y_{j}\right) ;$ and $R_{y x_{k, j}} \triangleq$ $E\left(y_{k} x_{j}\right)$. Those covariances have been derived in [4] (see Eq. (1.5-20)) as:

$$
\begin{aligned}
& R_{x x k, j}=R_{y y_{k, j}}=\frac{\sigma^{2} J_{0}\left(2 \pi F_{m} \tau_{k, j}\right)}{2\left[1+\left(\Delta \omega_{k, j} \sigma_{\tau}\right)^{2}\right]} \\
& R_{x y_{k, j}}=-R_{y x_{k, j}}=-\Delta \omega_{k, j} \sigma_{\tau} R_{x x k, j}
\end{aligned}
$$

where

$\sigma^{2}$ is the variance (power) of the complex Gaussian random processes ( $\frac{\sigma^{2}}{2}$ is the variance per each dimension).

$J_{0}$ is the zeroth-order Bessel function of the first kind.

$F_{m}$ is the maximum Doppler frequency $F_{m}=\frac{v}{\lambda}=\frac{v f_{c}}{c}$. In this formulas, $\lambda$ is the wavelength of the carrier, $f_{c}$ the carrier frequency, $c$ the speed of light and $v$ the mobile speed.

$\tau_{k, j}$ is the arrival time delay between two signals

$\Delta \omega_{k, j}=2 \pi\left(f_{k}-f_{j}\right)$ is the angular frequency separation between the two complex Gaussian processes with Rayleigh envelopes at frequencies $f_{k}$ and $f_{j}$.

$\sigma_{\tau}$ is the rms delay spread of the wireless channel.

The fading correlation properties between wireless channels as functions of antenna spacing in antenna arrays have been mentioned in [6]. Fig. 1 presents a typical model of the channel where all signals from a receiver are assumed to arrive at $T_{x}$ transmit antennas within $\pm \Delta$ at angle $\Phi(|\Phi| \leq \pi)$. Let $\lambda$ be the wavelength, $D$ the distance between the two adjacent transmit antennas, and $z=2 \pi \frac{D}{\lambda}$. Then, it has been proved that the closed-form expressions of the covariances normalized by the variance per each dimension (real and imaginary) are (see Eq. (A. 19) and (A. 20) in [6]):

$$
\begin{aligned}
\tilde{R_{x x k, j}} & =\tilde{R_{y y_{k, j}}}=J_{0}(z(k-j)) \\
& +2 \sum_{m=1}^{\infty} J_{2 m}(z(k-j)) \cos (2 m \Phi) \frac{\sin (2 m \Delta)}{2 m \Delta}(3) \\
\tilde{R_{x y}, j} & =-\tilde{R_{y x_{k, j}}}=2 \sum_{m=0}^{\infty}\left[J_{2 m+1}(z(k-j))\right. \\
& \left.\times \sin [(2 m+1) \Phi] \frac{\sin [(2 m+1) \Delta]}{(2 m+1) \Delta}\right]
\end{aligned}
$$

where $\tilde{R}_{k, j}=\frac{2 R_{k, j}}{\sigma^{2}}$. In other words, we have:

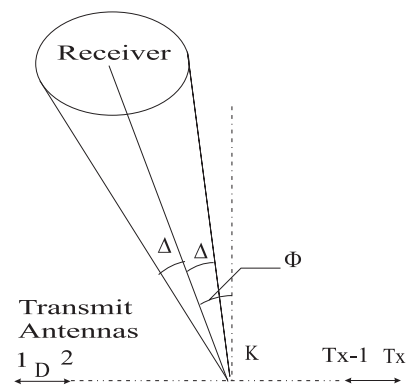

Figure 1. Model to examine the spatial correlation between transmit antennas.

$$
R_{k, j}=\frac{\sigma^{2} \tilde{R}_{k, j}}{2}
$$

$J_{q}$ is the first-kind Bessel function of the integer order $q$, and $\sigma^{2} / 2$ is the variance of the received signal at each transmit antenna per each dimension, i.e., it is assumed in [6] that the signals received from all transmit antennas have equal variances $\sigma^{2}$.

It should be emphasized that, the equalities (1) and (2) (similarly, (3) and (4)) hold only when the set of multi-path channel coefficients, which were denoted as $C_{n m}$ and derived in Eq. (1.5-1) and (1.5-2) in [4] (similarly, $g_{n}$ and Eq. (A-1) in [6]), as well as the powers are assumed to be the same for different random processes. Readers may refer to [4] (pp. 46-49) (similarly, [6] (pp. 1054-1056)) for an explicit exposition.

\section{Proposed generalized algorithm}

\subsection{Covariance matrix of complex Gaussian ran- dom variables with Rayleigh fading envelopes}

It is known that Rayleigh fading envelopes can be generated from the zero-mean complex Gaussian random variables. We consider here a column vector $\mathbb{Z}$ of $N$ zero-mean complex Gaussian random variables with variances $\sigma_{g_{j}}^{2}, j=1 \ldots N$. Denote $\mathbb{Z}=\left(z_{1}, \ldots, z_{N}\right)^{T}$, where $z_{j}$ $(j=1 \ldots N)$ is regarded as $z_{j}=r_{j} e^{i \theta_{j}}=x_{j}+i y_{j}$. It is assumed that the phases $\theta_{j}$ s are independent, identically uniformly distributed random variables. As a result, the real and imaginary parts of each $z_{j}$ are independent (but $z_{j} \mathrm{~s}$ are not necessarily independent), i.e., the covariances $E\left(x_{j} y_{j}\right)=0$ for $\forall j$ and therefore, $r_{j} \mathrm{~s}$ are Rayleigh envelopes. Let $\sigma_{g_{x j}}^{2}$ and $\sigma_{g_{y j}}^{2}$ be the variances per dimension (real and imaginary), i.e., $\sigma_{g_{x j}}^{2}=E\left(x_{j}^{2}\right), \sigma_{g_{y j}}^{2}=E\left(y_{j}^{2}\right)$. Clearly, $\sigma_{g_{j}}^{2}=\sigma_{g_{x j}}^{2}+\sigma_{g_{y j}}^{2}$. If $\sigma_{g_{x j}}^{2}=\sigma_{g_{y j}}^{2}$, then $\sigma_{g_{x j}}^{2}=\sigma_{g_{y j}}^{2}=\frac{\sigma_{g_{j}}^{2}}{2}$. Note that we consider a very general scenario where the variances (powers) of the real parts are not necessarily equal to those of the imaginary parts. Also, the powers of Rayleigh envelopes are not necessarily equal to one another. Therefore, the scenarios where the variances of those envelopes are equal to one another and the powers of real parts are equal to those of imaginary parts, such as those scenarios mentioned in Section 2, are considered as particular cases. For $k \neq j$, we define the covariances $R_{x x k, j}, R_{y y_{k, j}}, R_{x y_{k, j}}$ and $R_{y x} k, j$ between the 
real as well as imaginary parts of $z_{k}$ and $z_{j}$ similarly to those mentioned in Section 2. By definition, the covariance matrix $\mathcal{K}$ of $\mathbb{Z}$ is:

$$
\mathcal{K}=E\left(\mathbb{Z} \mathbb{Z}^{H}\right) \triangleq\left[\mu_{k, j}\right]_{N \times N}
$$

where $H$ denotes the Hermitian transposition operation and

$$
\begin{aligned}
& \mu_{k, j}= \\
& \begin{cases}\sigma_{g_{j}}^{2} & \text { if } k \equiv j \\
\left(R_{x x k, j}+R_{y y_{k, j}}\right)-i\left(R_{x y_{k, j}}-R_{y x k, j}\right) & \text { if } k \neq j\end{cases}
\end{aligned}
$$

\subsection{Forced positive semi-definiteness of the co- variance matrix}

First, we need to define the coloring matrix $\mathcal{L}$ corresponding to a covariance matrix $\mathcal{K}$. The coloring matrix $\mathcal{L}$ is defined to be the $N \times N$ matrix satisfying:

$$
\mathcal{L} \mathcal{L}^{H}=\mathcal{K}
$$

It is noted that the coloring matrix is not necessarily a lower triangular matrix. Particularly, to determine the coloring matrix $\mathcal{L}$ corresponding to a covariance matrix $\mathcal{K}$, we can use either Cholesky decomposition as mentioned in a number of papers, which have been reviewed in Section 1 of this paper, or eigen decomposition which is mentioned in the next section of this paper. The former yields a lower triangular coloring matrix, while the later yields a square coloring matrix.

Unlike determining $\mathcal{L}$ using Cholesky decomposition, where the covariance matrix $\mathcal{K}$ must be positive definite, determining $\mathcal{L}$ using eigen decomposition requires that $\mathcal{K}$ is at least positive semi-definite, i.e. the eigenvalues of $\mathcal{K}$ are either zeros or positive. We will explain later why the covariance matrix must be positive semi-definite even in the case where eigen decomposition is used to calculate the coloring matrix. The covariance matrix $\mathcal{K}$, in fact, may not be positive semi-definite.

To overcome this obstacle, similarly to (but not exactly as) the method in [7], we approximate the covariance matrix being not positive semi-definite by a positive semi-definite one. This procedure is presented as follows.

Assuming that $\mathcal{K}$ is the desired covariance matrix, which is not positive semi-definite. Perform the eigen decomposition $\mathcal{K}=\mathbf{V G V}^{H}$, where $\mathbf{V}$ is the matrix of eigenvectors and $\mathbf{G}$ is a diagonal matrix of eigenvalues of the matrix $\mathcal{K}$. Let $\mathbf{G}=\operatorname{diag}\left(\lambda_{1}, \ldots, \lambda_{N}\right)$. Calculate the approximate matrix $\boldsymbol{\Lambda} \triangleq \operatorname{diag}\left(\hat{\lambda}_{1}, \ldots, \hat{\lambda}_{N}\right)$, where:

$$
\hat{\lambda}_{j}= \begin{cases}\lambda_{j} & \text { if } \lambda_{j} \geq 0 \\ 0 & \text { if } \lambda_{j}<0\end{cases}
$$

We now compare our approximation procedure to the approximation procedure mentioned in [7]. The authors in [7] used the following approximation $\hat{\lambda}_{j}= \begin{cases}\lambda_{j} & \text { if } \lambda_{j}>0 \\ \varepsilon & \text { if } \lambda_{j} \leq 0\end{cases}$ where $\varepsilon$ is a small, positive real number.

Clearly, besides overcoming the disadvantage of Cholesky decomposition, our approximation procedure is more precise than the one mentioned in [7] since the matrix $\boldsymbol{\Lambda}$ in our algorithm approximates to the matrix $\mathbf{G}$ better than the one mentioned in [7]. Therefore, the desired covariance matrix $\mathcal{K}$ is well approximated by the positive semi-definite matrix $\mathbf{K}=\mathbf{V} \mathbf{\Lambda} \mathbf{V}^{H}$ from Frobenius point of view [7].

\subsection{Determine the coloring matrix using eigen de- composition}

In most of the conventional methods, except the one in [6], Cholesky decomposition was used to determine the coloring matrix. It has been well known that, Cholesky decomposition may not work for the covariance matrix which has eigenvalues being equal or close to zeros.

To overcome this disadvantage, similarly to (but not exactly as) the method proposed in [6], we use eigen decomposition, instead of Cholesky decomposition, to calculate the coloring matrix. It is important to note that estimating and comparing the computational efforts between the two methods (eigen decomposition versus Cholesky decomposition) are not our targets in this paper. The coloring matrix is calculated as follow:

At this stage, we have the forced positive semi-definite covariance matrix $\mathbf{K}$, which is equal to the desired covariance matrix $\mathcal{K}$ if $\mathcal{K}$ is positive semi-definite, or approximates to $\mathcal{K}$ otherwise. Further, as mentioned earlier, we have $\mathbf{K}=\mathbf{V} \boldsymbol{\Lambda} \mathbf{V}^{H}$, where $\boldsymbol{\Lambda}=\operatorname{diag}\left(\hat{\lambda}_{1}, \ldots, \hat{\lambda}_{N}\right)$ is the matrix of eigenvalues of $\mathbf{K}$. Since $\mathbf{K}$ is a positive semidefinite matrix, it follows that $\left\{\hat{\lambda}_{j}\right\}_{j=1}^{N}$ are real and nonnegative.

We now calculate a new matrix $\bar{\Lambda}$ as:

$$
\overline{\boldsymbol{\Lambda}}=\sqrt{\boldsymbol{\Lambda}}=\operatorname{diag}\left(\sqrt{\hat{\lambda}_{1}}, \ldots, \sqrt{\hat{\lambda}_{N}}\right)
$$

Clearly, $\bar{\Lambda}$ is a real, diagonal matrix that results in:

$$
\overline{\mathbf{\Lambda}} \overline{\mathbf{\Lambda}}^{H}=\overline{\mathbf{\Lambda}} \overline{\mathbf{\Lambda}}=\mathbf{\Lambda}
$$

If we denote $\mathbf{L} \triangleq \mathbf{V} \overline{\boldsymbol{\Lambda}}$, then it follows that:

$$
\mathbf{L} \mathbf{L}^{H}=\mathbf{V} \overline{\mathbf{\Lambda}} \overline{\boldsymbol{\Lambda}}^{H} \mathbf{V}^{H}=\mathbf{V} \boldsymbol{\Lambda} \mathbf{V}^{H}=\mathbf{K}
$$

It means that the coloring matrix $\mathbf{L}$ corresponding to the covariance matrix $\mathbf{K}$ can be computed without using Cholesky decomposition. Thereby, the shortcoming of the paper [7], which is related to round-off errors in Matlab caused by Cholesky decomposition, can be overcome.

We now explain why the covariance matrix must be positive semi-definite even when eigen decomposition is used to compute the coloring matrix. It is easy to realize that, if $\mathbf{K}$ is not positive semi-definite covariance matrix, then $\bar{\Lambda}$ calculated by (9) is a complex matrix. As a result, (10) and (11) are not satisfied.

\subsection{Proposed algorithm}

From the above observations, the authors propose here a generalized algorithm to generate $N$ correlated Rayleigh envelopes as given below:

1. In a general case, the desired variances (powers) $\left\{\sigma_{g_{j}}^{2}\right\}_{j=1}^{N}$ of complex Gaussian random variables with Rayleigh envelopes must be known. Specially, if one wants to generate Rayleigh envelopes corresponding to the desired variances (powers) $\left\{\sigma_{r_{j}}^{2}\right\}_{j=1}^{N}$, then $\left\{\sigma_{g_{j}}^{2}\right\}_{j=1}^{N}$ are calculated as follows:

$$
\sigma_{g_{j}}^{2}=\frac{\sigma_{r j}^{2}}{\left(1-\frac{\pi}{4}\right)} \quad \forall j=1 \ldots N
$$


2. From the desired correlation properties of correlated complex Gaussian random variables with Rayleigh envelopes, determine the covariances $R_{x x_{k, j}}, R_{y y_{k, j}}, R_{x y_{k, j}}$ and $R_{y x_{k, j}}$, for $k, j=1, \ldots, N$ and $k \neq j$. In other words, in a general case, those covariances must be known.

Specially, in the case where the powers of all random processes are equal and other conditions hold as mentioned in Section 2, we can follow equations (1) and (2) in the case of time delay and frequency separation, such as in OFDM systems, or equations (3), (4) and (5) in the case of spatial separation like with antenna arrays in MIMO systems to calculate the covariances $R_{x x k, j}, R_{y y_{k, j}}, R_{x y_{k, j}}$ and $R_{y x_{k, j}}$.

The values $\left\{\sigma_{g_{j}}^{2}\right\}_{j=1}^{N}, R_{x x k, j}, R_{y y_{k, j}}, R_{x y_{k, j}}$ and $R_{y x_{k, j}}(k, j=1, \ldots, N ; k \neq j)$ are the input data of our proposed algorithm.

3. Create the $N \times N$-sized covariance matrix $\mathcal{K}$ following (6) and (7). The covariance matrix of complex Gaussian random variables is considered here, as opposed to the covariance matrix of Rayleigh fading envelopes like in the conventional methods.

4. Perform the eigen decomposition $\mathcal{K}=\mathbf{V G V}^{H}$. Denote $\mathbf{G} \triangleq \operatorname{diag}\left(\lambda_{1}, \ldots, \lambda_{N}\right)$. Then, calculate a new diagonal matrix $\boldsymbol{\Lambda}=\operatorname{diag}\left(\hat{\lambda}_{1}, \ldots, \hat{\lambda}_{N}\right)$, where $\hat{\lambda}_{j}$ are calculated following (8). Thereby, we have a diagonal matrix $\boldsymbol{\Lambda}$ with all elements in the main diagonal being real and definitely non-negative.

5. Determine a new matrix $\bar{\Lambda}=\sqrt{\Lambda}$ and calculate the coloring matrix $\mathbf{L}$ by setting $\mathbf{L}=\mathbf{V} \bar{\Lambda}$.

6. Generate a column vector $\mathbb{W}$ of $N$ independent complex Gaussian random samples with zero means and arbitrary, equal variances $\sigma_{g}^{2}: \mathbb{W}=\left(u_{1}, \ldots, u_{N}\right)^{T}$.

7. Generate a column vector $\mathbb{Z}$ of $N$ correlated complex Gaussian random samples as follows $\mathbb{Z}=\frac{\mathbf{L} \mathbb{W}}{\sigma_{g}} \triangleq$ $\left(z_{1} \ldots, z_{N}\right)^{T}$. The elements $\left\{z_{j}\right\}_{j=1}^{N}$ are zero-mean, correlated, complex Gaussian random variables with variances $\left\{\sigma_{g}^{2}\right\}_{j=1}^{N}$. The $N$ moduli $\left\{r_{j}\right\}_{j=1}^{N}$ of the Gaussian samples in $\mathbb{Z}$ are the desired Rayleigh fading envelopes.

\section{Generation of correlated Rayleigh en- velopes in a real-time scenario}

In Section 3.4, we have proposed the algorithm for generating $N$ correlated Rayleigh fading envelopes in multipath, flat fading channels in a single time instant. We can repeat steps 6 and 7 of this algorithm to generate Rayleigh envelopes in the continuous time interval. It is noted that, the discrete-time samples of each Rayleigh fading process generated by this algorithm in different time instants are independent of one another.

It has been known that the discrete-time samples of each realistic Rayleigh fading process may have autocorrelation properties, which are the functions of the Doppler frequency

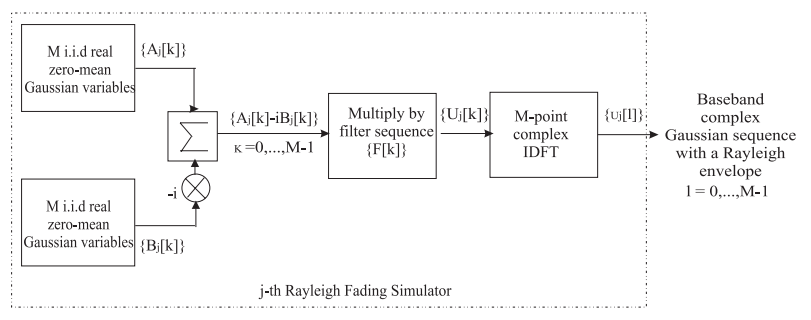

\section{Figure 2. Model of a Rayleigh generator for an individual Rayleigh envelope.}

corresponding to the motion of receivers as well as other factors such as the sampling frequency of transmitted signals. It is because the band-limited communication channels not only limit the bandwidth of transmitted signals, but also limit the bandwidth of fading. This filtering effect limits the rate of changes of fading in time domain, and consequently, results in the autocorrelation properties of fading. Therefore, the algorithm generating Rayleigh fading envelopes in realistic conditions must consider the autocorrelation properties of Rayleigh fading envelopes.

To simulate a realistic multipath fading channel, Doppler filters are normally used. The most recent computerassisted model providing an accurate channel realization was proposed by Young in [8]. It should be emphasized that, in [8], the model is aimed at generating an individual Rayleigh envelope corresponding to a certain normalized autocorrelation function of itself, rather than generating different Rayleigh envelopes corresponding to a desired covariance matrix (autocorrelation and cross-correlation properties between those envelopes).

Therefore, the model for generating $N$ correlated Rayleigh fading envelopes in realistic fading channels (each individual envelope is corresponding to a desired normalized autocorrelation property) can be created by associating the model proposed in [8] with our algorithm mentioned in Section 3.4 in such a way that, the resultant Rayleigh fading envelopes are corresponding to the desired covariance matrix.

This combination must overcome the main shortcoming of the method proposed in [7] as analyzed in Section 1. This is an easy task in our algorithm. The key for the success of this task is the modification in steps 6 and 7 of our algorithm (see Section 3.4), where the variances of $N$ complex Gaussian random variables are not fixed as in [7], but can be arbitrary in our algorithm. Again, besides being more generalized, our modification in these steps allows the $a c$ curate combination of the method proposed in [8] and our algorithm, i.e. guaranteeing that the generated Rayleigh envelopes are exactly corresponding to the desired covariance matrix.

The model of a Rayleigh fading generator for generating an individual baseband Rayleigh fading envelope corresponding to a desired normalized autocorrelation function proposed in [8] is shown in Fig. 2. This model generates a Rayleigh fading envelope using Inverse Discrete Fourier Transform (IDFT), based on independent zero-mean Gaussian random variables weighted by appropriate Doppler filter coefficients. The sequence $\left\{u_{j}[l]\right\}_{l=0}^{M-1}$ of the complex Gaussian random samples at the output of the $j^{\text {th }}$ Rayleigh generator (Fig. 2) can be expressed as: $u_{j}[l]=$ $\frac{1}{M} \sum_{k=0}^{M-1} U_{j}[k] e^{i \frac{2 \pi k l}{M}}$ where: 
$M$ denotes the number of points with which the IDFT is carried out;

$l$ is the discrete-time sample index $(l=0, \ldots, M-1)$; $U_{j}[k]=F[k] A_{j}[k]-i F[k] B_{j}[k]$;

$\{F[k]\}$ are the Doppler filter coefficients.

For brevity, we omit the subscript $j$ in the expressions, except when this subscript is necessary to emphasize. If we denote $u[l]=u_{R}[l]+i u_{I}[l]$, then it has been proved that, the autocorrelation property between the real parts $u_{R}[l]$ and $u_{R}[m]$ as well as that between the imaginary parts $u_{I}[l]$ and $u_{I}[m]$ at different discrete-time instants $l$ and $m$ is as given below (see Eq. (7) in [8]):

$$
\begin{aligned}
r_{R R}[l, m] & =r_{I I}[l, m]=r_{R R}[d]=r_{I I}[d] \\
& =E\left\{u_{R}[l] u_{R}[m]\right\}=\frac{\sigma_{\text {orig }}^{2}}{M} \operatorname{Re}\{g[d]\}(13)
\end{aligned}
$$

where $d \triangleq l-m$ is the sample lag, $\sigma_{\text {orig }}^{2}$ is the variance of the real, independent zero-mean Gaussian random sequences $\{A[k]\}$ and $\{B[k]\}$ at the inputs of Doppler filters, and the sequence $\{g[d]\}$ is the IDFT of $\left\{F[k]^{2}\right\}$, i.e.:

$$
g[d]=\frac{1}{M} \sum_{k=0}^{M-1} F[k]^{2} e^{i \frac{2 \pi k d}{M}}
$$

Similarly, the correlation property between the real part $u_{R}[l]$ and the imaginary part $u_{I}[m]$ is calculated as (see Eq. (8) in [8]):

$$
r_{R I}[d]=E\left\{u_{R}[l] u_{I}[m]\right\}=\frac{\sigma_{\text {orig }}^{2}}{M} \operatorname{Im}\{g[d]\}
$$

The mean value of the output sequence $\{u[l]\}$ has been proved to be zero (see Appendix A in [8]). have:

If $d=0$ and $\{F[k]\}$ are real, from (13), (14) and (15), we

$$
\begin{aligned}
& r_{R R}[0]=r_{I I}[0]=E\left\{u_{R}[l] u_{R}[l]\right\}=\frac{\sigma_{\text {orig }}^{2}}{M^{2}} \sum_{k=0}^{M-1} F[k]^{2} \\
& r_{R I}[0]=E\left\{u_{R}[l] u_{I}[l]\right\}=0
\end{aligned}
$$

Therefore, by definition, the variance of the sequence $\{u[l]\}$ at the output of the Rayleigh generator is:

$$
\begin{aligned}
\sigma_{g}^{2} & \triangleq E\left\{u[l] u[l]^{*}\right\} \\
& =2 E\left\{u_{R}[l] u_{R}[l]\right\}=\frac{2 \sigma_{\text {orig }}^{2}}{M^{2}} \sum_{k=0}^{M-1} F[k]^{2}
\end{aligned}
$$

where ${ }^{*}$ denotes the complex conjugate operation.

Let $J_{0}\left(2 \pi f_{m} d\right)$ be the function:

$$
J_{0}\left(2 \pi f_{m} d\right)=\frac{r_{R R}[d]}{\sigma_{g}^{2}}=\frac{r_{I I}[d]}{\sigma_{g}^{2}}
$$

i.e. $J_{0}\left(2 \pi f_{m} d\right)$ be the autocorrelation function in (13) normalized by the variance $\sigma_{g}^{2}$ in (16). In the above equation, $J_{0}$ denotes the zero-order Bessel function of the first kind and $f_{m}$ is the maximum Doppler frequency $F_{m}$ normalized by the sampling frequency $F_{s}$ of the transmitted signals, i.e.
$f_{m}=\frac{F_{m}}{F_{s}} . J_{0}\left(2 \pi f_{m} d\right)$ is called the normalized autocorrelation function.

To achieve a desired normalized autocorrelation function $J_{0}\left(2 \pi f_{m} d\right)$, the Doppler filter $\{F[k]\}$ is determined in Young's model [8] as given below (see Eq. (21) in [8]).

$$
F[k]= \begin{cases}0 & \mathrm{k}=0 \\ \sqrt{\frac{1}{2 \sqrt{1-\left(\frac{k}{M f m}\right)^{2}}}} & \mathrm{k}=1, \ldots, k_{m}-1 \\ \sqrt{\frac{k_{m}}{2}\left[\frac{\pi}{2}-\arctan \left(\frac{k_{m}-1}{\sqrt{2 k_{m}-1}}\right)\right]} & \mathrm{k}=k_{m} \\ 0 & \mathrm{k}=k_{m}+1, \ldots, \mathrm{M}-k_{m}-1 \\ \sqrt{\frac{k_{m}}{2}\left[\frac{\pi}{2}-\arctan \left(\frac{k_{m}-1}{\sqrt{2 k_{m}-1}}\right)\right]} & \mathrm{k}=\mathrm{M}-k_{m} \\ \sqrt{\frac{1}{2 \sqrt{1-\left(\frac{M-k}{M f m}\right)^{2}}}} & \mathrm{k}=\mathrm{M}-k_{m}+1, \ldots, \mathrm{M}-1\end{cases}
$$

In (18), $k_{m} \triangleq\left\lfloor f_{m} M\right\rfloor$, where $\lfloor$.$\rfloor indicates the biggest$ rounded integer being less or equal to the argument.

It has been proved in [8] that the (real) filter coefficients in (18) will produce a complex Gaussian sequence with the normalized autocorrelation function $J_{0}\left(2 \pi f_{m} d\right)$, where $d$ is the sample lag, and with the expected independence between the real and imaginary parts of Gaussian samples, i.e., the correlation property in (15) is zero. The zerocorrelation property between the real and imaginary parts is necessary in order that the resultant envelopes are Rayleigh distributed.

To generate $N$ correlated Rayleigh fading envelopes in a real-time scenario where Doppler frequency shifts, $N$ Rayleigh generators, each of which is presented in Fig. 2, are simultaneously used. To generate $N$ correlated Rayleigh envelopes corresponding to a desired covariance matrix at an observed discrete-time instant $l(l=$ $0, \ldots, M-1)$, similarly to the method in [7], we take the output $u_{j}[l]$ of the $j^{t h}$ Rayleigh simulator, for $j=$ $1, \ldots, N$, and input it as the element $u_{j}$ into step 6 of our algorithm proposed in Section 3.4. However, as opposed to the method in [7], the variance $\sigma_{g}^{2}$ of complex Gaussian samples $u_{j}$ in step 6 of our method is calculated following (16). This value is used as the input parameter for steps 6 and 7 of our algorithm. Thereby, the variance-changing effect caused by Doppler filters is taken into consideration in our algorithm, and consequently, our proposed algorithm overcomes the main shortcoming of the method in [7].

The algorithm for generating $N$ correlated Rayleigh envelopes (when Doppler frequency shifts are considered) $a t$ a discrete-time instant $l$, for $l=0, \ldots, M-1$, can be summarized as:

1. Perform the steps 1 to 5 mentioned in Section 3.4.

2. From the desired autocorrelation properties (13) and (17) of each of the complex Gaussian random sequences (with Rayleigh fading envelopes), determine the desired value $M$. The value of $M$ is also the number of points with which IDFT is carried out.

3. For each Rayleigh generator presented in Fig. 2, generate $M$ complex Gaussian random variables $\{A[k]-$ $i B[k]\}$ with zero means and variances $\sigma_{\text {orig }}^{2}$ per dimension. 


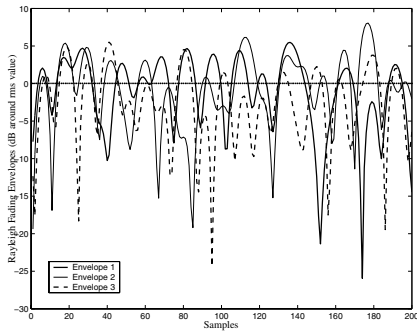

(a) Spectral correlation

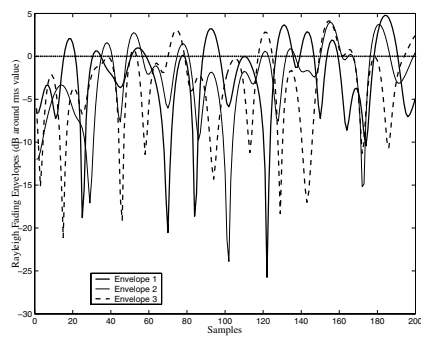

(b) Spatial correlation

\section{Figure 3. Examples of three equal power cor- related Rayleigh fading envelopes.}

4. Multiply complex Gaussian samples $\{A[k]-i B[k]\}$, for $k=1, \ldots, M$, with the corresponding filter coefficient $F[k]$ given in (18).

5. Perform $M$-point IDFT of the resultant samples.

6. Calculate the variance $\sigma_{g}^{2}$ of the output $\{u[l]\}$ following (16). It is noted that $\sigma_{g}^{2}$ is the same for $N$ Rayleigh generators. We also emphasize that, by this calculation, the variance-changing effect of Doppler filters are taken into account.

7. Generate a column vector $\mathbb{W}=\left(u_{1}, \ldots, u_{N}\right)^{T}$ of $N$ i.i.d. complex Gaussian random samples with the distribution $\left(0, \sigma_{g}^{2}\right)$ where the element $u_{j}$, for $j=$ $1, \ldots, N$, is the output $u_{j}[l]$ of the $j^{t h}$ Rayleigh generator and $\sigma_{g}^{2}$ has been calculated in step 6 .

8. Continue the step 7 mentioned in Section 3.4. The $N$ envelopes of elements in the column vector $\mathbb{Z}$ are the desired Rayleigh envelopes at the considered time instant $l$.

Steps 7 and 8 are repeated for different time instants $l(l=$ $0, \ldots, M-1)$, and therefore, the algorithm can be used for a real-time scenario.

\section{Simulation results}

In this section, first, we simulate $N=3$ frequencycorrelated Rayleigh fading envelopes corresponding to the complex Gaussian random variables with equal powers $\sigma_{g_{j}}^{2}=1(j=1, \ldots, 3)$ in the flat fading channels. Parameters considered here include $M=4096$ (the number of IDFT points), $\sigma_{\text {orig }}^{2}=1 / 2$ (variances per dimension in Young's model), $F_{s}=1 \mathrm{kHz}, F_{m}=50 \mathrm{~Hz}$ (corresponding to a carrier frequency $900 \mathrm{MHz}$ and a mobile speed $v=60$ $\mathrm{km} / \mathrm{hr}$ ). Therefore, we have $f_{m}=0.05, k_{m}=204$. Frequency separation between two adjacent carrier frequencies considered here is $200 \mathrm{kHz}$ (e.g in GSM 900) and we assume that $f_{1}>f_{2}>f_{3}$. Also, we consider the rms delay spread $\sigma_{\tau}=1 \mu \mathrm{s}$ and time delays between three envelopes are $\tau_{1,2}=1 \mathrm{~ms}, \tau_{2,3}=3 \mathrm{~ms}, \tau_{1,3}=4 \mathrm{~ms}$.
From (1), (2), (6) and (7), we have the desired covariance matrix $\mathcal{K}$ as given below:

$$
\left[\begin{array}{ccc}
1 & 0.3782+0.4753 i & 0.0878+0.2207 i \\
0.3782-0.4753 i & 1 & 0.3063+0.3849 i \\
0.0878-0.2207 i & 0.3063-0.3849 i & 1
\end{array}\right]
$$

It is easy to check that $\mathcal{K}$ in (19) is positive definite. Using the proposed algorithm in Section 4, we have the simulation result presented in Fig. 3(a).

Next, we simulate $N=3$ spatially correlated Rayleigh fading envelopes. We consider an antenna array comprising three transmitter antennas, which are equally separated by a distance $D$. Assume that $\frac{D}{\lambda}=1$, i.e., $D=33.3 \mathrm{~cm}$ for GSM 900. Additionally, we assume that $\Delta=\pi / 18 \mathrm{rad}$ (or $\left.\Delta=10^{\circ}\right)$ and $\Phi=0 \mathrm{rad}$. The parameters $\sigma_{g_{j}}^{2}$ and $\sigma_{\text {orig }}^{2}$ are the same as the previous case.

From (3), (4), (5), (6) and (7), we have the following desired covariance matrix:

$$
\left[\begin{array}{ccc}
1 & 0.8123 & 0.3730 \\
0.8123 & 1 & 0.8123 \\
0.3730 & 0.8123 & 1
\end{array}\right]
$$

Since $\Phi=0 \mathrm{rad}$, the covariances $R_{x y_{k, j}}$ and $R_{y x_{k, j}}$ between the real and imaginary components of any pair of the complex Gaussian random processes (with Rayleigh fading envelopes) are zeros, and consequently, $\mathcal{K}$ is a real matrix. Readers may refer to (4) and (5) for more details. It is easy to realize that $\mathcal{K}$ in $(20)$ is positive definite. The simulation result is presented in Fig. 3(b).

\section{Conclusion}

In this paper, we have derived a more generalized algorithm to generate correlated Rayleigh fading envelopes, which is applicable to various scenarios. Besides being more generalized, our proposed algorithm (with or without Doppler spectrum spread) is more precise, while overcoming all shortcomings of the conventional methods.

\section{References}

[1] N. C. Beaulieu. Generation of correlated Rayleigh fading envelopes. IEEE Commun. Lett., 3(6):172-174, June 1999.

[2] N. C. Beaulieu and M. L. Merani. Efficient simulation of correlated diversity channels. Proc. IEEE Wireless Communications and Networking Conference WCNC 2000, 1:207-210, Sept. 2000.

[3] R. B. Ertel and J. H. Reed. Generation of two equal power correlated Rayleigh fading envelopes. IEEE Commun. Lett., 2(10):276-278, Oct. 1998.

[4] W. C. Jakes. Microwave mobile communications. John Wiley \& Sons, New York, 1974.

[5] B. Natarajan, C. R. Nassar, and V. Chandrasekhar. Generation of correlated Rayleigh fading envelopes for spread spectrum applications. IEEE Commun. Lett., 4(1):9-11, Jan. 2000.

[6] J. Salz and J. H. Winters. Effect of fading correlation on adaptive arrays in digital mobile radio. IEEE Trans. Veh. Technol., 43(4):1049-1057, Nov. 1994.

[7] S. Sorooshyari and D. G. Daut. Generation of correlated Rayleigh fading envelopes for accurate performance analysis of diversity systems. Proc. 14th IEEE Int. Symp. Personal, Indoor and Mobile Radio Commun., 2:1800-1804, Sept. 2003.

[8] D. J. Young and N. C. Beaulieu. The generation of correlated Rayleigh random variates by inverse discrete Fourier transform. IEEE Trans. Commun., 48(7):1114-1127, July 2000. 